Fig. 19-38. Fixirung bei $100-110^{\circ} \mathrm{C}$. Färbang mit dem Laurent'schen Farbstoffe.

Fig. 19-22. Eosinophile Zellen mit versch. gefärbten Granulis.

Fig. 19. Färbg. mit heisser Lösung; Differenzirung mit Anilinöl-Xylol kurz. Fig. 20,21.

Fig. 22 . $" \quad "$ Mischung;

Fig. 23-38. P'seudo-eosin. Zellè.

Fig. 23. Lösung heiss; Differenzirung kurz; spärliche einfarbige Granula mit Beziehungen zu Fäden.

Fig. 24, 25. Lösụng heiss; Differenzirung läng; spärliche blaue u. zahlreiche ungefarbte Granula.

Fig. 26, 27. Lösung kalt; Differenzirung kurz; verschieden gefärbte Granula; Beziehungen zu Fäden; event. nur die grösseren Granula blau.

Fig. 28, 29. Lösung kalt; Differenzirung lang; spärliche rothe feine Granula; event. Beziehungen zu Fäden.

Fig. 30. Miscbung heiss; Differenzirung kurz; versehiedenfarbige Granula; Beziehungen der basophil. Körnchen zu Kern- oder Zellwand?

Fig. 31-38. Lösung kalt mit Ueberschuss von Methylenblau.

Fig. 31-36. Bei kurzer Differenzirung.

Fig. 37, 38. " langer ,

\title{
XV.
}

\section{Ueber das Auftreten von Myelin in Zellen und seine Beziehung zur Fettmetamorphose.}

(Aus dem Pathologischen Institut zu Berlin.)

$$
\text { Von }
$$

Dr. med. C. Kaiserling,

Privatdocenten und Assistenten am Pathologischen Institut in Berlin und

Dr. Arnold Orgler, prakt. Arzt in Berlin.

Schon im Jahre 1895 war es Kaiserling gelegentlich der Untersuchungen über die Fixirbarkeit der verschiedenen Zellen und Zelleinschlüsse aufgefallen, dass in der Nebennieren-Rinde unter den als Fett bezeichneten Tropfen ein Unterschied bestand, derart, dass der weit grössere Theil dieser Tropfen bei der An- 
wendung polarisirten Liehtes sich als doppeltbrechend erwies. Die genauere Analyse diéser Elemente wurde von A. Orgle ${ }^{1}$ vorgenommen, dessen Resultate zum Theil in desseñ InauguralDissertation, Berlin 1898, niedergelegt sind. Die Grösse der in Rede stehenden Kugeln ist eine sehr verschiedene. Sie schwankt zwischen 0,4 und etwa $18 \mu$, doch pflegen die meisten im Durchschnitt 4-6 $\mu$ zu messen. Gegen Natronlauge und Essigsäure sind sie widerstandsfähig, ebenso gegen Schwefelsäure. In Chloroform und Aether sind sie leicht, in Alkohol schwerer löslich. Mit Osmiumsäure färb̈en sie sich nur leicht grau, niemals so intensiv wie Fett, mit Sudan III und Scharlach R roth. Die osmirten Präparate entfärben sich im Gegensatz zu Fett mit Xylol, Chloroform und Bergamottöl. Bei allen diesen Untersuchungen verlieren die Körnchen ihre charakteristische Eigenschaft der Doppelbrechung, und wer nicht seine Versuche so anstellt, dass er nur doppelbrechende Elemente in seinen Präparaten hat, oder doch wenigstens durch andere Merkmale, wie Gruppirung, Nachbarschaft unveränderlicher Objecte u. dergl. für eine nachträgliche sichere Identificirung sorgt, kann leicht zu Verwechslungen mit wirklichen Fetttropfen verleitet werden... Der Verlust der Doppelbrechung tritt auch bei allen von uns versuchten Härtungs-Methoden ein. Daher kann an fixirten Präparaten über diese doppelbrechenden Körnchen kein Aufschluss erlangt werden. Nur am frischen Präparat ohne irgend einen differenten Zusatz muss man untersuchen, am besten an feinen Doppelmesser-Schnitten. Jeder Zusatz schafft ein Kunstproduct. Schon das einfache Eintrocknen eines Abstrich-Präparates z. B. von der Nebennieren-Rinde führt neben der groben Gestalt-Veränderung der zumeist kreisiunden Elemente zum Verluste der Doppelbrechung. In diesem Falle gelingt es durch Wasserzusatz nicht nur wieder kugelige Gebilde, sondern auch ihre Doppelbrechung wieder herzustellen. Leider ist etwas Aehnliches uns bei den herkömmlichen Fixirungs-Methoden nicht gelungen. Hier bleibt wohl die Form erhalten, soweit nicht etwa Lösung eintritt, wie beim Alkohol, aber nicht die Doppelbrechung, und es ist nachträglich ganz unmöglich, die sichere Unterscheidung von Fett zu behaupten. Diese Umstände machen die genaue Untersuchung auch in chemischer Beziehung zu einer sehr 
schwierigen und zweifelhaften. Wir werden darauf weiter unten zurückkommen. Jedenfalls mahnt aber auch diese Thatsache zu grosser Vorsicht gegenüber den an fixirten und mit complicirten Behandlungs- und Färbungs-Methoden gewonnenen Resultaten.

Wir haben bei unseren Untersuchungen stets mit einer einfachen Polarisations-Einrichtung von Carl Zeiss, Jena, gearbeitet. Das Polarisations-Prisma wird in den Blendenträger des $A b b$ e'schen BeleuchtungsApparates eingehängt.' Oberhalb des Polarisators werden die genau passenden, in einer Pappscheibe befestigten Gips- und Quarzplättchen aufgelegt. Für viele Untersuchungen ist ein genau centrirter, drehbarer Objecttisch nicht zu entbehren. Der Analysator wird auf das Ocular aufgesetzt und gestattet mittels einfacher Gradtheilung die Ablesung des Drehungswinkels. Für die einstweilen für histologische Zwecke erwünschte Untersuchung genügt diese einfache Einrichtung und man kannn der complicirten, wenn auch ungleich leistungsfähigeren Einrichtung des speciell für diese Untersuchung construirten Polarisations-Mikroskopes entbehren. Nach Abnahme des Analysators kann man auch bei eingehängtem Polarisator in gewohnter Weise mikroskopiren und hat zur Untersuchung auf Doppelbrechung nur nöthig, den Analysator anfzusetzen. Die durch den Polarisator bewirkte erhebliche Beeinträehtigung der Apertur des Abbe'schen Beleuchtungs" Apparates ist bei der Untersuchung frischer Objecte belanglos, da sie ja ohnehin eine starke Abblendung des Beleuchtungs-Kegels erfordern. Sebr bequem ist die neuere Einrichtung des herausklappbaren Condensors von Zeiss, der mit einem einzigen Handgriffe die Entfernung der BeleuchtungsLinsen und Benutzung der Oylinder-Irisblende gestattet. Dass man bei der Untersuchung frischer Objecte immer ohne Beleuchtungs-Linsen arbeiten soll, ist eine Uebertreibung und nur bei ganz schwachen Objectiven zu empfehlen. Durch geeignete Abblendung und eventuelle Verwendung etwas schiefer Beleuchtung lassen sich alle Feinheiten eines farblosen Objectes darstellen. Am deutlichsten wird die Doppelbrechung bei gekreuzten Nikols, wo auf dunklem Grunde die betreffenden anisotropen Gegenstände hell aufleuchten. Bei schwachen Vergrösserungen stört das von oben auf das Präparat auffallende Licht. Damit man sich vor Irrthümern schützt, empfehlen wir eine dunkle Umhällung des Objectivs bis zum Object. Meist genügt das Vorbalten der Hand. Eventuell controlirt man in zweifelhaften Fällen durch Einschaltung eines entsprechenden Gipsplättchens. Die Technik der Untersuchung ist bei Dippel (2) und Ambronn (3) hinreichend ausführlich beschrieben.

Betrachtet man nun einen geeigneten frischen Schnitt durch die Nebennieren-Rinde bei mittlerer Vergrösserung, so werden die doppeltbrechenden Kugeln schon bei hellem Gesichtsfelde, also bei parallel liegenden Schwingungs-Ebenen von Polarisator und Analysator kenntlich durch einen 
schwaehen scbwärzlichen Schatten, der den sichtbaren optischen Querschnitt derselben in vier gleiche Quadranten theilt. Bei gekreuzten PolarisationsEbenen erscheint das ganze Gesichtsfeld dunkel, nur die anisotropen Kugeln leuchten hell in weisser Farbe und zeigen ein intensives schwarzes Kreuz. Nach Einschaltung eines Gipsplättchens Roth I. Ordnung erscheint, wenn seine längere Achse mit der Schwingungs-Ebene des Polarisators einen Winkel von $45^{\circ}$ bildet, bei gekreuzten Nikols das vorher dunkele Gesichtsfeld in einem bestimmten Roth, je zwei gegenüberliegende vorher helle Felder hingegen in blauer, die beiden anderen in gelber Farbe. Wie daraus nähere Schlüsse auf die Lage, der Elasticitäts-Achsen gezogen werden können, ist bei orgler (l. c.) nachzusehen.

Während wir nun anfangs annahmen, dass diese doppelbrechenden Körnchen ein specifisches Product der NebennierenRinde wären, da sie uns in den zahlreichen anderen untersuchten Organen nicht begegnet waren, fand $K$ aiserling sie weiterhin in einer Amylö̈-Niere mit starker Fettmetamorphose. Hier war beinahe Alles, was bisher als Fett-Tropfen angesehen wurde, doppelbrechend. Fett im menschlichen Körper haben wir nie diese 'Eigenschaft besitzen sehen. Also konnte, auch das in den Nieren vorhandene doppelbrechende Material kein Fett im landläufigen Sinne sein. Wir kamen nun auf Grund verschiedener Erwägungen dahin, 'zu untersuchen, ob diese doppelbrechenden Körner etwa auch in anderen Fällen vorhanden seien, wo man bisher eine Fettmetamorphose annahm. Wir untersuchten daraufhin eine grosse Anzahl von Organen. Zunächst schien uns die Thymusdrüse dazu geeignet, deren Function bald nach der Geburt aufhört und die zum Theil mit Fettmetamorphose zu Grunde geht. Es wurde eine grosse Anzahl von Drüsen untersucht, die dann weiterhin von Orgler zu chemischen Analysen im chemischen Laboratorium des Pathologischen Instituts unter Salkowski's Leitung verarbeitet wurden. Ueber diese Untersuchungen ist in der vorhergehenden Arbeit berichtet. Bei Neugeborenen fanden sich die doppelbrechenden Körnchen nie, während sie bei älteren Kindern mit zunehmender Rückbildung der Drüse immer zahlreicher auftraten, sodass schliesslich die betreffenden Zellen vollständig mit doppelbrechenden Tropfen durchsetzt waren und keine Structur, Kerne u.dergl. mehr erkennen liessen. Sie boten völlig das Bild der sogenannten Körnchen-Kagel dar.

Ein weiteres Beispiel der Fettmetamorphose liefert das Herz. Bei den untersuchten Fällen fand sich niemals, weder in Anfangs- 
stadien noch in den vorgeschrittenen Formen ein anisotropes Körnchen, sondern alles Fett gleicht dem gewöhnlichen Körperfett, sowohI in seinen optischen Eigenschaften, wie auch gegenüber den mikrochemischen Reactionen, der Färbung mit Sudan und Scharlach, der Fähigkeit, sich mit Osmiumsäure zu schwärzen und die Schwärzung bei Behandlung mit Xylol, Chloroform ünd Bergamottöl zu behalten. Die neueren Untersuchungen von Ribbert ${ }^{4}$, Göbel ${ }^{5}$, Rosenfeld ${ }^{6}$ haben es sehr wahrscheinlich gemacht, dass es sich bei den Funden von Fett in der HerzMusculatur nicht um eine Degeneration im alten Sinne, d. h. eine Bildung von Fett aus den Eiweiss-Substanzen der Zelle, sondern mehr um eine Infiltration, eine Aufnahme von Fett aus den Fettlagern des Körpers handelt. Auch unsere Untersuchungen zeigen einen Unterschied dieser Fettanhäufung gegenüber der bei der regressiven Metamorphose der Thymusdrüse.

Ganz anders verhalten sich die Zellen der Imtima der Arterien, vornehmlich der Aorta. Bei der Fettmetamorphose der Intimazellen ist ein sehr erheblicher Theil der Tropfen doppelbrechend. Zieht man die innerste Schicht einer geeigneten Aorta, welche das makroskopisch schon so auffallende feinbestaubte Aussehen darbietet ohne schwerere Veränderun- . gen atheromatöser oder sclerotischer Processe, möglichst vorsichtig und dünn $a b$, so erhält man die elegantesten Bilder, welche bei diesen Untersuchungen uns begegnet sind. Die spindel- und sternförmigen Zellen sind mit grösseren und kleineren Kugeln in mehr oder weniger grosser Ausdehnung erfüllt, mitunter in Körnchenzellen umgewandelt und im dunkeln Felde bei gekreuzten Nikols leuchten alle diese Kugeln hell auf und zeigen das schwarze Kreuz. Dabei sei bemerkt, dass die elastischen Fasern selber doppelbrechend sind. Die Grösse der Kugeln in den einzelnen Zellen ist eine sehr wechselnde, man findet kleine und grosse, stark und schwächer doppeltbrechende neben einander, und in den grossen sternförmigen Zellen ist nicht selten von den Details kaum noch etwas zu sehen, da die übereinander gehäuften Kugeln gegenseitig die Lichtverhältnisse so stören, dass man nur einen hellen Haufen erkennt. Daneben kommt besonders bei complicirten Fällen auch gewöhnliches Fett in Tropfenform vor. 
Das Vorkommen doppeltbrechender Körnchen in den fettig metamorphosirten Zellen der Amyloidnieren ist schon erwähnt. Sie liegen in mehr oder minder grosser Zahl in den Epithelien der gewundenen Harncanälchen. Aehnlich war das Verhalten bei der grossen weissen Niere. Hier fanden wir zuerst einen völligen Zerfall der Epithelzellen und Uebergang der Körnchen in das Lumen der Harnkanälchen. In anderen Fällen waren die Epithelien in grösserem Zusammenhange gelöst und lagen als Fettkörnchen-Cylinder frei im Lumen der Harncanälchen. Bei einem hierher gehörigen Falle waren die FettkörnchenCylinder auch im trüben Urin der Blase zu finden und erwiesen sich ebenfalls als doppeltbrechend. Endlich gelang es auch sie im Harne eines an Bright'scher Krankheit leidenden $\mathrm{Pa}$ tienten bei dessen Lebzeiten nachzuweisen. Bei Granularatrophie der Nieren und acuten Fällen von parenchymatöser Nephritis insbesondere bei Infectionskrankheiten, Scharlach, Diphtherie u. s. w. fanden wir sie nicht, sondern hier war alles vorhandene Fett isotrop. Auch bei experimenteller Phosphor-Vergiftung an Kaninchen war das Fett der Epithelien das gewöhnliche.

Sehr häufig begegnet man den doppelbrechenden Tropfen im Schleim der Bronchien und deren Epithelzellen in AbstrichPräparaten. Bei der fibrinösen Lungenentzündung finden sie sich sehr reichlich. Wir haben sie ferner in den fettig metamorphosirten Epithelzellen bei chronischen Bronchopneumonien and bei tuberculösen Processen gefunden. Erst nach Abschluss der vorliegenden Arbeit stiessen wir auf die Untersuchungen von Schmidt ${ }^{7}$, Müller ${ }^{8}$, deren unverfänglicher Titel Niemanden vermuthen lassen konnte, dass sie von den gleichen Tropfen handelten. Namentlich aus der Arbeit Müller's, überschrieben "Zusatz zu vorstehender Arbeit", geht hervor, dass die in der "vorstehenden" Arbeit von A. Schmidt, über Herkunft und chemische Natur der Myelinformen des Sputums beschriebenen Tropfen mit den von uns vielfach nachgewiesenen doppeltbrechenden Körnern identisch sind. Schmidt ist der Ansicht, dass sie ${ }_{n}$ nur im Bereiche der Luftröhren- und Bronchialschleimhaut, d.h. im Secrete derselben vorkommen". "Dass es (das Myelin) wirklich als solches von der Schleimhaut des. Bronchialbaumes abgesondert wird, dafür liegen ausser den erwähnten verschollten Epithel- 
zellen, von denen erst noch nachgewiesen werden muss, dass sie mit Myelín imbibirt sind, keine greifbaren Anhaltspunkte vor. Es ist wohl denkbar, dass Myelintropfen erst im Secret selbst gebildet werden..." Wir sind geneigt, alle diese Myelintropfen. als die Reste zerfallenerEpithelien anzusehen, da sie in Hunderten. von Fällen in den Zellen vorkommen.

Es giebt noch ein sehr gutes Beispiel für den Zusammenhang des Auftretens unserer Körnchen mit regressiven Veränderungen der Zellen, ihr Vorkommen im Corpus luteum der Ovarien, wo sie besonders schön in der Form der Körnchenkugel auftreten. Bei einigermassen vorgeschrittener Rückbildung findet man sie auch oft in solchen Fällen frei im Präparate, aber das dürfte durch die Zertrümmerung der Körnchenzellen beim Schneiden verursacht werden. Ein einwandfreies Auftreten dieser Körnchen ausserhallb der Zellen haben wir nicht zu finden vermocht, möchten vielmehr zu der Ansicht neigen, dass in diesen Fällen stets eine Zertrümmerung oder wie im Sputum und Urin eine Auflösung von ursprünglichen Zellen stattgefunden hat. Dazu möchten wir auch das wiederholte Auftreten im Nebennieren-Venenblute rechnen, da nach den Untersuchungen $\mathrm{Ma}$ nasse's ' der Zusammenhang der Nebennieren-Zellen mit den Venen ein sehr inniger und die Nebenniere gegen mechanische Einwirkungen ungemein empfindlich ist. Besonders die Rindenzellen mit ihren doppeltbrechenden Körnern zertrümmern selbst bei vorsichtiger Präparation leicht, und wenn man sie bei derSection gar mit Fingern anfasst; zertrümmert man sicher solche Zellen und findet dann ihren Inhalt auch im Blute oder extracellulär im Gewebe.

Häufig begegneten wir den doppeltbrechenden Körnchen in Geschwülsten. Sehr zahlreich pflegen sie in den erweichten. und fettig metamorphosirten Geschwulstzellen von NebennierenTumoren zu sein. Hier kommen neben typischen Körnchenzellen auch andere Zellen vor, die eine schwache diffuse Doppelbrechung wahrnehmen liessen. Ueber die Bedeutung letzteren Befundes vermögen wir einstweilen noch nichts Genaues anzugeben. Vielleicht lässt sich aber ein sehr häufiges Vorkommen doppelbrechender Körnchenzellen zu einer Entscheidung verwenden, $o b$ eine zweifelhafte Geschwulst der Niere oder der Nebennieren- 


\section{3}

Nachbarschaft von der Nebenniere oder versprengten! Keimen dieses Organs ausgeht. Sie fanden sich sowohl bei Cárinom wie bei Sarkom der Nebenniere. Dass ihnen aber kèin besonderer diagnostischer Werth zukommt, beweist das Vorkommen der anisotropen Körnchen in anderen Tumoren. Sehr gute Bilder liefern oft ganz kleine Metastasen. Bei einem solchen Präparate von einer krebsigen Lebermetastase, bei dessen Anfertigung der Schnitt durch die Mitte des wenig mehr als miliaren Knotens geführt war, sahen wir, dass alle peripherisch gelegenen Zellen frei von fettähnlichen Tropfen waren, während die vier bis fünf central gelegenen Zellen feine Tropfen enthielten, die bei gekreuzten Nikols das charakteristische Aussehen der doppeltbrechenden Körnchen darboten. Die in der Leber vorhandenen Tropfen waren isotrop. Es sind aber keineswegs alle in den Geschwulstzellen vorkommenden Tropfen doppeltbrechend, sondern in vielen Zellen ist wirkliches Fett vorhanden und selbst in solchen Fällen, wo mikroskopisch eine Fettmetamorphose vorhanden ist, sind häufig keine oder nur verschwindend wenig doppeltbrechende Körnchen vorhanden. Auch in Sarkomen haben wir diese Körnchen wiederholt beobachtet.

Dass die doppeltbrechenden Körner bei der sogenannten Fettmetamorphose des Herzens fehlen, ist bereits erwähnt. Ebenso haben wir sie vermisst bei der Fettinfiltration der Leber, in den normalen Fettdepets des Körpers, im Colostrum, der secernirenden Milchdrüse und vielen Fällen von Fettmetamorphose der Nieren.

So einfach es für einen in der Untersuchung frischen Materials geübten Beobachter ist, das Vorhandensein oder Fehlen der doppeltbrechenden Substanz nachzuweisen und die scharf von isotropen Fettpartikeln zu unterscheiden, so schwierig ist es oft, die weiteren Verhältnisse, die Kernbeschaffenheit u. s. w. der Zellen zu prüfen. Es giebt wenig Fälle, wo nur anisotrope Tropfen auftreten. Mitunter in derselben Zelle, meist aber in anderen benachbarten Zellen finden sich gewöhnliche Fettsubstanzen. Da durch die üblichen Fixationsmethoden die Doppelbrechung verloren geht, so ist nachträglich keine sichere Unterscheidung mehr möglich. Eine blosse Vermuthung, selbst eine hohe Wahrscheinlichkeit der Identität ist kein Beweis. Daher 
sind wir zu einem völligen Abschluss dieser Untersuchungen noch nicht gekommen. In den Nebennieren sind die Kerne oft völlig gut erhalten und färbbar, so dass wir keinen Anhaltspunkt dafür haben, ob das Auftreten doppeltbrechender Kugeln mit degenerativen Processen im Zusammenhang stehe und neigen eher der Ansicht zu, dass das Gegentheil der Fall ist. Anders aber verhält es sich in den übrigen Fällen. Hier scheint in der That beim Auftreten dieser Kugeln die Zelle in ihren normalen Verhältnissen mehr oder weniger geschädigt zu sein und in mehreren Fällen ist bei den Körnchenzellen mit Sicherheit ein Fehlen des Kernes und protoplasmatischer $Z$ wischensubstanzen festzustellen. Besonders ist dies der Fall bei den Nierenepithelien, den betreffenden. Zellen aus Lunge und Corpus luteum, sowie den Intimazellen der Aorta. Weitere Untersuchungen sind im Gange.

Was sind nun diese anisotropen Kugeln und was bedeuten sie im Haushalte der Zellen? Da die mikrochemischen Reactionen, die uns einstweilen zu Gebote stehen, nicht genügen, so haben wir versucht, auch durch chemische Untersuchungen Aufschluss zu erhalten. Orgler hat diesen Theil der Arbeit ausgeführt und in der vorstehenden Arbeit darüber berichtet. Leider war uns die Veröffentlichung von Schmidt und Müller entgangen. Ihnen ist es gelungen, aus den Myelin-Kugeln des Sputum Protagon darzustellen, einen Körper, der eine höchst complicirte Zusammensetzung hat, aber kein Fett ist. Wir verstehen unter Fett ein Gemenge von Tripalmitin, Tristearin und Triolein. Es giebt verschiedene Arten von Fett, aber sie unterscheiden sich nur durch die Mischungs-Verhältnisse dieser drei Componenten. Protagon hingegen besteht im Wesentlichen aus Cerebrin und Lecithin, oder sagen wir lieber, diese beiden Körper lassen sich aus Protagon abspalten. Das Cerebrin ist ein noch nicht genau chemisch definirter COHN-haltiger Körper, das Lecithin nach den Untersuchungen von P. Bergell ein Distearin-GlycerinPhosphorsäurecholin. Man kann also das Protagon weder ein Fett noch einen fettähnlichen Körper nennen, so wenig wie man von den Eiweisskörpern als kohlehydrat-ähnlichen Substanzen spricht, weil sie eine Kohlehydrat-Gruppe enthalten. Da nun häufig in der Mikrochemie die zu untersuchende Substanz durch 
eine Gruppen-Reaction bestimmt wird - beim Nachweis des Fettes durch Osmiumsäure ist z. B. die Oelsäure die vorwiegend reagirende Componente -, so läuft man Gefahr, Stoffe verschiedener Zusammensetzung zu einer Klasse zu vereinigen, die zwar die betreffende Gruppen-Reaction geben, sonst aber nichts miteinander zu thun haben. Hier kann nur die gründliche Untersuchung mit den chemischen Methoden aushelfen. Aber auch die chemischen Methoden sind so tief eingreifend, dass durch sie mit Sicherheit allerlei Veränderungen in der molecularen Beschaffenheit der ursprünglichen Rohstoffe hervorgerufen werden. Zum Mindesten geben sie uns nur eine sehr unvollkommene Aufklärung über die Natur des Rohmaterials. Wenn man sich das vergegenwärtigt, wird es verständlich, wie z. B. aus den Nebennieren die verschiedensten Dinge durch verschiedene Untersucher dargestellt wurden. So erzeugte F. und S. Marino-Zucco ${ }^{10}$ und seine Mitarbeiter Guarnieri"1 ${ }^{11}$ und S. Marino-Zucco ${ }^{12}$ aus dem Aether-Extract der Nebennieren Neurin, Alexander ${ }^{13}$ Lecithin, ähnlich Manasse ${ }^{14}$. Andere waren bestrebt, die wirksame Substanz darzustellen, der man die Blutdruck steigernde Wirkung von Nebennieren-Extracten zuzuschreiben habe. So erhielt S. Fränkel ${ }^{15}$ ein N-haltiges Orthodioxybenzol-Derivat, F ürth ${ }^{16}$ ein Dioxypyridin.

Was nun auch die doppeltbrechenden Körper in der Nebennieren-Rinde sein mögen, als Fett oder fettähnliche Körper können wir sie nicht bezeichnen. Ehe wir nicht ihre Natur genauer kennen, wollen wir auch von der Erfindung eines neuen Namens, der den Anschein erweckt, als ob wir mit ihm einen wohldefinirten chemischen Begriff verbinden könnten, absehen und den für ähnliche morphologische Elemente seit Virchow üblichen, auch von Müller und Schmidt beibehaltenen Namen Myelin gebrauchen. Nur insofern möchten wir diesen Begriff etwas genauer definirt wissen, als wir darunter die in Zellen meist als Kugeln auftretende, doppeltbrechende Substanz verstehen, wie sie oben geschildert ist.

In den Nebennieren fanden wir diese Myelinkugeln bei Kindern fast ausschliesslich, bei älteren Individuen treten noch andere, Tettropfen ähnliche Gebilde auf. Von diesen ist ein Theil durch Osmiumsäure schwarz zu färben, isotrop, ein anderer 
Theil zwar auch färbbar, aber gegen Lösungsmittel wènig resistent. Im frischen Präparat ist noch eine Gruppe auffallend, die nur an den Rändern der Segmente einen geringen Grad von Anisotropie zeigt, während das schwarze Kreuz fast den ganzen übrigen Theil der Kugel einnimmt. Wir haben trotz vieler Versuche bis jetzt keine Möglichkeit finden können, die Beziehungen dè drei Gruppen von Tropfen zu einander und ihre chemische Beschaffenheit feststellen zu können.

Ebenso schwierig ist es, über die Entstehung der doppeltbrechenden Kugeln ein sicheres Urtheil zu finden. Da wir sie nur in einigen Fällen schwerer Abzehrung bei chronischen Phthisikern in der Nebennieren-Rinde vermisst, sonst aber stets in allen Lebensaltern gefunden haben, scheint uns ihr Auftreten in den Nebennieren ein physiologisches und normales zu sein. Dafür spricht auch die Erhaltung der Zellstructur. Wie sie in die Zellen hineinkommen, können wir vorläufig auch nicht sagen, da wir bei der morphologischen Betrachtung immer nur einen fertigen Zustand vor Augen haben, von dem nicht einmal zu sagen ist, ob er nicht nach dem Tode gegenüber dem im Lebenden eine Veränderung exlitten hat. Da wir die MyelinKugeln auch an frisch exstirpirten, sofort unter allen Vorsichtsmassregeln auf erwärmtem Objectträger untersuchten Nebennieren ebenfalls gefunden haben, so dïrfte ihr Vorhandensein in der lebenden Zelle eine gewisse Wahrscheinlichkeit für sich haben.

Nun ist es möglich, dass die Substanz, aus der sie bestehen, als solche in die Zellen eintritt und nach Art der Fett-Infiltration an anderen Zellen nur aufgespeichert wird, oder aber, dass sie erst in der Zelle aufgebaut wird. Sehr wahrscheinlich besteht eine nähere Verwandtschaft dieses Myelins zu gewissen Substanzen des Hirns und des Nerven, als zum Fett. Aber die Untersuchungen, welche von Lomer ${ }^{17}$, Weigert ${ }^{18}$, Z ander ${ }^{19}$, Alexander ${ }^{20}$ über die Beziehungen der Hirn-Entwicklung und der der Nebennieren angestellt wurden, haben keine befriedigende Lösung der Frage gegeben. Wenn der Eine eine Abhängigkeit der Hirn-Entwicklung von der der Nebennieren gefunden zu haben glaubt, leugnet sie der Andere, und der Dritte glaubt genau das Umgekehrte, während in der Feststellung der Thatsachen keine wesentlichen Verschiedenheiten bestehen. Hier 
müssen neue Untersuchungen vorwiegend experimenteller Natur angestellt werden. Die. Verwerthung von Missbildungen ist immer nur mit grösster Vorsicht zu gebrauchen, ehe bewiesen ist, dass das gleichzeitige Bestehen verschiedener Missbildungen auch wirklich ein causales ist. Jedenfalls ist die Lösung dieser Frage ein wichtiges Glied in dem Beweise, ob das Vorkommen des Myelins in der Nebenniere als eine Generation, oder als eine Infiltration anzusehen ist. Einstweilen erscheint es uns wahrscheinlich, dass das Myelin in den Zellen der NebennierenRinde aufgebaut wird. Hierzu müssen wir die Vorgänge in anderen Organen vergleichend heranziehen. Dazu eignet sich vorzugsweise die Thymus-Drüse, in deren Zellen erst nach der Geburt beim Beginne der Rückbildung die Myelin-Kugeln auftreten. Durch die chemischen Untersuchungen von A. Orgler (a. a. 0.) hat sich ergeben, dass in den Thymus-Drüsen Neugeborener der Aether-Alkohol-Ex tract nach Menge und PhosphorGehalt gleich ist dem der myelinhaltigen, regressiv sich ändernden Drüse. Daraus kann man schliessen, dass das Auftreten von Myelin ohne ein nachweisbares Hinzukommen anderer Stoffe geschieht, als ursprünglich in der noch functionirenden Drüse vorhanden waren. Nur die moleculare Structur einzelner. Verbindungen ändert sich, oder es treten Umlagerungen und Spaltungen gewisser Gruppen ein. Alle diese Stoffe waren also vorhanden in der Zellsubstanz. Vermeidet man den Namen Protoplasma; aus dem sich bei vielen damn sofort die Vorstellung entwickelt, als handele es sich um Eiweiss, und nennt die morphologisch nicht näher definirten Stoffe in der Zelle einfach Zellsubstanz, so hat man in der Thymus das, was Virchow seinerzeit als Fett-Metamorphose beschrieb. Nur war man damals nicht in der Lage, die als Tropfen in den Zellen auftretenden fettähnlichen Dinge genau zu trennen und kannte auch die grosse Mannigfaltigkeit der Zellsubstanzen noch nicht genau, sondern hielt sie vorwiegend für Eiweiss, was ja damals mehr morphologisch als chemisch definirt wurde. So entstand die Definition: Fett-Metamorphose ist die Umwandlung von Eiweiss in Fett. Neuere Untersuchungen haben, wie Lubarsch ${ }^{21}$ in einem zusammenfassenden Artikel darlegt, dazu geführt, diese lange Zeit unangetastete Lehre völlig zu erschüttern und alles 
Auftreten von Fett beziehungsweise von Substanzen, die in den Alkohol-Aether-Extract übergehen, in den Zellen als infiltrirte, aus den Fettdepots des Körpers stammende Fettstoffe zu erklären. Für viele Fälle sogenannter Fett-Metamorphose mag das gelten, so für die Fett-Metamorphose der Herzmusculatur, bei PhosphorVergiftung, bei der Milchsecretion und manchen nephritischen Zuständen. Aber auch hier bleibt zu untersuchen, ob nicht etwa das Auftreten von Fett nur ein Sichtbarwerden, ein Ausscheiden vorher gelösten, zum Zellbestand gehörigen Fettes oder fettähnlicher Körper ist. In sehr vielen dieser Fälle ist eine Schädigung der Zellstructur nachweisbar, in manchen Fällen dürfte es sich um die Anfangsstadien einer vorerst nur functionellen Störung handeln. Wenn man die Fett-Infiltration für einen normalen physiologischen Zustand erklärt, so in den Fett-Gewebszellen, den Leberzellen, so muss man diejenigen Erscheinungsformen der Fett-Infiltration von diesen trennen, wo die Infiltration in Zellen auftritt, deren Aufgabe eine andere ist, als Fett aufzuspeichern, wie mir das von den Herzmuskel-Zellen, den Nieren-Epithelien u. s. w. der Fall zu sein scheint. Das sind ebensowenig normale Zustände, wie die übertrieben starke Infiltration der Leberzellen bei Abzehrungs-Krankheiten, z. B. bei Phthisis pulmonum. Zum Mindesten sind das functionelle Abweichungen. Dafür sprechen die Untersuchungen Rosenfeld's ${ }^{22}$, der eine Wechselbeziehung zwischen dem Auftreten von Fett und dem Schwinden von Glykogen in den Zellen nachgewiesen hat. In allen diesen drei Zuständen des Fett-Auftretens fanden wir kein Myelin. Die Formen, bei denen Myelin auftritt, müssen von den vorstehend erörterten ganz streng getrennt werden, da die Aehnlichkeit der Tropfen mit Fett nur eine rein morphologische ist, während es sich gar nicht um Fett handelt. Im Gegensatze zu den Nebennieren erscheinen die übrigen von uns untersuchten Zellen sich im Zustand der Nekrobiose zu befinden. Vielleicht bestehen hier noch ebenso viele Gruppen, wie bei der Fett-Infiltration. Bis alle diese Verhältnisse geklärt sind, schlagen wir für die ausserhalb der Nebenniere vorkommenden Zell-Veränderungen mit Auftreten von Myelin den Namen myelinogene Nekrobiose oder entsprechend der FettMetamorphose den Namen Myelin-Metamorphose vor, da uns 
die Kriterien Virchow's für Fett-Metamorphose für diese Form zuzutreffen erscheinen mit der Modification, dass statt Eiweiss Zellsubstanz, statt Fett Myelin gesetzt wird. Natürlich ist die vorgeschlagene Bezeichnung nur eine provisorische, bis die chemische Natur des sog. Myelins genau erkannt ist und die Vorgänge in den Zellen genauer studirt sind. Aus diesem Grunde sehen wir von irgend einer Neubenennung ab und wir sind schon selber an der Arbeit, Myelin und myelinhaltige Zellen weiter zu analysiren. Ebenso bleibt zu prüfen, was aus dem Myelin weiterhin wird. Ein Theil desselben wird als solches im Sputum, im Harn ausgeschieden, der weitaus grösste Theil dürfte jedoch im Organismus weiter verbraucht werden. Bei allen diesen Untersuchungen stösst man Schritt auf Schritt auf Schwierigkeiten, die nicht nur in dem Material und den Untersuchungsmethoden liegen, sondern auch im Beobachter selber. Es ist sehr verführerisch und auch oft üblich, aus einem gleichzeitigen Vorkommen von Substanzen auf einen causalen $\mathrm{Zu}$ sammenhang zu schliessen und aus einer verschiedenen Intensität nebeneinander bestehender Zustände ein consecutives Verhältniss und aus den Zuständen todten Materials einen im Leben verlaufenden Process zu construiren, sich durch eine plausible Theorie selber zu binden und teleologische Erwägungen ảnzustellen. Je weniger man alles das sich selber gestattet, umso mehr wachsen die Schwierigkeiten. Dazu kommt, dass dem einzelnen Beobachter nicht alle erwünschten Hilfsmittel zu Gebote stehen, über die ein anderer verfügt. Aus diesem Grunde theilen wir unsere Beobachtungen mit und hoffen, das sich Untersucher finden werden, auf diesem Gebiete weiter zu arbeiten, auf einem Gebiete, wo fast noch alle Fragen der endgültigen Lösung harren. Das ist aber nicht möglich durch die Discussion bestehender Thatsachen, sondern durch Auffindung so vieler neuer, dass nur eine Lösung des Problems - was ist Myelin, wie entsteht es, welchen Zweck hat es? - möglich ist.

\section{Literatur.}

1. Orgler: Inaugural-Dissertation. Berlin 1898.

2. Dippel: Das Mikroskop und seine Anwendung. Bd. I. Braunschweig 1882. 
3. Ambronn: Anleitung zur. Benutzung des Polarisationsmikroskops bei histologischen Untersuchungen.

4. Rib bert: Dieses Archiv. Bd. 147.

5. Göbel: Centralblatt für allgemeine Pathologie und pathologische Anatomie. 1893.

6. Rosenfeld: Centralblatt für innere Medicin. 1901, No. 6.

7. Schmidt: Berliner klinische Wochenschrift. 1897, No. 4.

8. Fr. M üller: Ebenda. 1897, No. 4.

9. Manasse: Dieses Archiv. Bd. 135.

10. F. Marino-Zuceo: Chemisches Centralblatt. 1888.

11. F. Marino-Zucco und Guarineri: Ebenda. 1888.

12. F. Marino-Zucco und S. Marino-Zucco: Moleschott's Untersuchungen zur Naturlehre des Menschen und der Thiere. Bd: 15.

13. A lexander: Ziegler's Beiträge. Bd. 11.

14. Manasse: Zeitschrift für physiologische Chemie. Bd. 20.

15. S. Fränkel: Wiener medicinische Blätter. 1896, No. 14 ff.

16. Fürth: Zeitschrift für physiologische Chemie. Bd. 25.

17. Lomer: Dieses Archiv. Bd. 98.

18. Weigert: Ebenda. Bd. 100.

19. Zander: Ziegler's Beiträge. Bd. 7.

20. Alexander: a. a. 0 .

21. Lubarsch: Lubarsch und Ostertag Ergebnisse u. s. w. 1897.

22. Ros enfeld: Ueber Organverfettungen, Verhandlungen des 19. Congresses für innere Medicin, Berlin 1901.

\section{XVI.}

\section{Ueber den Fettgehalt normaler und in regres- siver Metamorphose befindlicher Thymusdrüsen.}

(Aus dem chemischen Laboratorium des Pathologisehen Instituts der Universität Berlin.

Von

Dr. Arnold Orgler, Arzt in Berlin.

Die mikroskopischen Untersuchungen, die Kaiserling und ich in der vorhergehenden Arbeit veröffentlicht haben, zeigen, dass in einer Anzahl von pathologisehen Veränderungen, die man bis jetzt mit dem Ausdruck Fett-Metamorphose oder fettige Degeneration bezeichnete, fettähnliche Tröpfchen auftreten, die sich durch 